\title{
Imaging of neuroinflammation: TSPO and beyond
}

\author{
Andrea Varrone $^{1} \cdot$ Adriaan A. Lammertsma $^{2}$
}

Received: 3 October 2015/Accepted: 12 October 2015/Published online: 29 October 2015

(C) Italian Association of Nuclear Medicine and Molecular Imaging 2015

Neuroinflammation is a pathological phenomenon common to many disorders of the central nervous system (CNS). The process includes activation of microglia, the resident immune cells of the CNS, production of both pro- and antiinflammatory mediators, tissue damage, tissue repair, and activation of astrocytes. This entire spectrum of phenomena represents the way in which the CNS reacts to any kind of insult, either acute or chronic. Since neuroinflammation can have both detrimental and beneficial effects, knowledge of the relative contributions of these two actions of the immune cells of the CNS could provide a means to selectively intervene in specific inflammatory processes and thus modify the detrimental outcome that can lead to tissue damage or neurodegeneration.

The chemical and cellular mediators of inflammation (cytokines, microglia, astrocytes, myeloid cells, T-cells) [1], the phenotypic characterization of pro- and anti-inflammatory microglia and other specific receptors, and targets of inflammation (for instance toll-like receptors, purinergic receptors, fractalkine receptors) are known and well-studied, both in vitro and using animal models of neuroinflammation.

However, at present, most of the inflammatory processes occurring in vivo cannot be studied in the living brain.

Positron emission tomography (PET) has made it possible to examine in vivo the $18-\mathrm{kDa}$ translocator protein (TSPO) as a marker of microglia activation and neuroinflammation in a variety of CNS disorders, such as stroke,

Andrea Varrone

andrea.varrone@ki.se

1 Department of Clinical Neuroscience, Centre for Psychiatry Research, Karolinska Institutet, Stockholm, Sweden

2 Department of Radiology \& Nuclear Medicine, VU University Medical Center, Amsterdam, The Netherlands multiple sclerosis, Parkinson's disease, Multiple System Atrophy, Progressive Supranuclear Palsy, Alzheimer's disease, and other dementias. For several reasons, however, TSPO imaging is not the "Holy Grail" for studying neuroinflammation in vivo. First, quantification of TSPO availability using the reference tracer $\left[{ }^{11} \mathrm{C}\right]-R$-PK11195 is complex and requires advanced methods with several assumptions regarding the physiological behavior of the radioligand. Second, the development of second-generation TSPO radioligands only in part has provided improved imaging tools, as the presence of low-, mixed-, and highaffinity binders contributes to large variability in the data. Third, it is well known that TSPO is not a specific marker for activated microglia, since it is also expressed on astrocytes. Finally, TSPO does not permit a distinction between pro- and anti-inflammatory microglia, which is a serious limitation when considering possible therapeutical strategies.

The knowhow acquired over the last 15-20 years on advantages and limitations of TSPO imaging as a marker of neuroinflammation underscores the need for new alternative neuroinflammation imaging markers. A tool complementary to TSPO imaging is the measurement of monoamino oxidase B (MAO-B) activity using $\left[{ }^{11} \mathrm{C}\right]-\mathrm{L}-$ Deprenyl-D2 PET. There clearly is an imbalance between the large number of studies performed using TSPO radioligands and the limited number of studies using MAO-B radioligands as imaging tools of neuroinflammation. Therefore, at present, the complementary value of MAO-B imaging can still not be assessed adequately using available data. In addition, to the best of our knowledge, there is no human study that has compared TSPO and MAO-B imaging in any CNS disorders, so it is still difficult to make any comments on the relative value of the two markers for imaging neuroinflammation. 
The European Union Concerted Action "Imaging of Neuroinflammation in Neurodegeneration" (INMiND) was established and funded by the 7th Framework Programme in order to examine different aspects of neuroinflammation in neurodegenerative and other CNS disorders, using a translational approach ranging from target identification, through rodent models of CNS disorders to neuroinflammation in human subjects [2]. One of the goals of the Consortium is to develop new radioligands for imaging other targets of neuroinflammation, such as $\mathrm{P} 2 \mathrm{X} 7$ receptors, metalloproteinases, and $\mathrm{CB} 2$ receptors that could be useful complementary targets to the well-established TSPO and MAO-B.

Is there a need for a third generation of TSPO radioligands? Our view is that the third generation of radioligands for neuroinflammation will probably include targets other than TSPO. The examination of new targets specific for either pro-inflammatory or anti-inflammatory phenotypes of microglia will most likely provide additional knowledge on the role of these immune cells in the regulation of neuroinflammation. This knowledge will be pivotal in the design of new therapeutical strategies that modify the neuroinflammatory cascade [3] in order to reduce detrimental effects and promote anti-inflammatory processes that lead to tissue repair. In the future, we hope to see studies that will combine imaging of TSPO with third generation radioligands to understand how microglia are involved in detrimental and beneficial effects in CNS disorders.

Author's contribution Andrea Varrone and Adriaan A. Lammertsma: literature search and review, content planning manuscript writing, and editing.

\section{Compliance with ethics standards}

Conflict of interest Andrea Varrone and Adriaan A. Lammertsma declare no conflicts of interest.

Research involving human participants and/or animals This article does not contain any studies with human participants performed by any of the authors.

\section{References}

1. Ransohoff RM, Schafer D, Vincent A, Blachère NE, Bar-Or A (2015) Neuroinflammation: ways in which the immune system affects the brain. Neurotherapeutics 12(4):896-909. doi:10.1007/ s13311-015-0385-3

2. Mohammadi D (2013) INMiND: getting to the bottom of neuroinflammation. Lancet Neurol 12(12):1135-1136. doi:10. 1016/S1474-4422(13)70268-0

3. Heneka MT, Kummer MP, Latz E (2014) Innate immune activation in neurodegenerative disease. Nat Rev Immunol 14(7):463-477. doi:10.1038/nri3705 